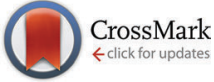

Cite this: Phys. Chem. Chem. Phys., 2015, 17, 15598

Received 15th December 2014 Accepted 27th March 2015

DOI: $10.1039 / c 4 c p 05877 a$

www.rsc.org/pccp

\title{
Specific adhesion of membranes simultaneously supports dual heterogeneities in lipids and proteins $\dagger$
}

\author{
O. Shindell, N. Mica, M. Ritzer and V. D. Gordon*
}

\begin{abstract}
Membrane adhesion is a vital component of many biological processes. Heterogeneities in lipid and protein composition are often associated with the adhesion site. These heterogeneities are thought to play functional roles in facilitating signalling. Here we experimentally examine this phenomenon using model membranes made of a mixture of lipids that is near a phase boundary at room temperature. Non-adherent model membranes are in a well-mixed, disordered-fluid lipid phase indicated by homogeneous distribution of a fluorescent dye that is a marker for the fluid-disordered $\left(L_{d}\right)$ phase. We specifically adhere membranes to a flat substrate bilayer using biotin-avidin binding. Adhesion produces two types of coexisting heterogeneities: an ordered lipid phase that excludes binding proteins and the fluorescent membrane dye, and a disordered lipid phase that is enriched in both binding proteins and membrane dye compared with the non-adhered portion of the same membrane. Thus, a single type of adhesion interaction (biotin-avidin binding), in an initially-homogeneous system, simultaneously stabilizes both ordered-phase and disordered-phase heterogeneities that are compositionally distinct from the non-adhered portion of the vesicle. These heterogeneities are long-lived and unchanged upon increased temperature.
\end{abstract}

\section{Introduction}

Adhesion of a biological membrane to another membrane or an extracellular matrix substrate is a widespread phenomenon in biology. Membrane adhesion is requisite for many vital biological processes, including exocytosis, intracellular trafficking, and sexual reproduction. Heterogeneities in the lipid and protein composition of the membrane are often associated with adhesion sites; these heterogeneities are frequently known as "rafts" and are thought to facilitate signalling by clustering together proteins with an affinity for the liquid-ordered lipid phase that characterizes the raft. ${ }^{1-6}$ To elucidate the biophysical principles undergirding raft formation, experimental physical scientists have often used simplified experimental model systems in the form of giant unilamellar vesicles (GUVs) that are typically made of a high-melting lipid, a low-melting lipid, and a sterol. Such biophysical studies have largely focused on lipid phase separation at thermodynamic equilibrium, ${ }^{7-13}$ and on the effects of biologically-relevant perturbations such as tension ${ }^{12,13}$ and curvature and undulations. ${ }^{11,14}$ We have previously shown that

The University of Texas at Austin, Department of Physics and Center for Nonlinear Dynamics, 2515 Speedway, Stop C1610, Austin, Texas 78712-1199, USA.

E-mail: gordon@chaos.utexas.edu

$\dagger$ Electronic supplementary information (ESI) available. See DOI: 10.1039/ c4cp05877a non-specific membrane adhesion can promote the formation of an ordered lipid phase. ${ }^{11}$

Traditionally, specific adhesion of membranes via protein-like binders ${ }^{15-19}$ has been studied separately from lipid phase separation. Specific adhesion can produce heterogeneities in binding-protein distribution via equilibrium and kinetic mechanisms. It has recently been shown that, for ternary-mixture GUVs near a critical point, adhesion can stabilize compositional heterogeneities that result from the molecular affinity of the biotin-conjugated lipids, used to control binding, for the fluorescent dye that marks the disordered lipid phase. ${ }^{20}$ However, we are far from a comprehensive understanding of the interactions of adhesion with membrane heterogeneities. This is complicated even further by cases such as the immunological synapse, in which more than one type of compositional heterogeneity is simultaneously stabilized by adhesion. ${ }^{21-25}$ Understanding and controlling the interactions of adhesion and biomembrane heterogeneities will advance our understanding of fundamental biological processes and could lead to new therapeutic pathways that rectify abnormalities in adhesion-related signalling. It also will advance possibilities for making "smart," responsive membrane-based containers for technological use.

Here we show that membrane adhesion that is mediated by only one binding species can simultaneously support two different types of heterogeneity - an internal ordered-phase lipid domain surrounded by a peripheral disordered-phase lipid domain. 
Binding proteins are localized to the disordered-phase region of the adhesion site, which is also enriched in the disorderedphase dye compared with the non-adhered, disordered-phase portion of the membrane. The rough annular patterns that adhesion produces are stable over the timescale of hours up to a day. Thus, we show that a single type of adhesive interaction can sort membrane components into two types of long-lived, heterogeneous structures. Moreover, we show that some features of these heterogeneities could be out of equilibrium. This is important, because there are more possibilities for tuning the properties of membrane heterogeneities if out-of-equilibrium states are accessible.

\section{Materials}

The lipids used in this study were purchased from Avanti Polar Lipids: 1,2-dioleoyl-sn-glycero-3-phosphocholine (DOPC); 1,2-dipalmitoyl-sn-glycero-3-phosphocholine (DPPC); 1,2-diphytanoylsn-glycero-3-phosphocholine (DiPhyPC); cholesterol (Chol); 1,2-dioleoyl-sn-glycero-3-phosphoethanolamine- $N$-(cap biotinyl) (sodium salt) (biotin-DOPE); and 1,2-dioleoyl-sn-glycero-3-phosphoethanolamine- $N$-[methoxy(polyethylene glycol)-2000] (ammonium salt) (PEG-DOPE). PEG-2000 has a radius of 1.2-1.6 nm measured by small-angle neutron scattering. ${ }^{26}$ Binding proteins and dyes were purchased from Invitrogen: BODIPY ${ }^{\circledR}$ Fluorescent membrane dye (Bodipy); Avidin, NeutrAvidin ${ }^{\circledR}$ Biotin-binding Protein (neutravidin); Avidin, NeutrAvidin ${ }^{\circledR}$, Oregon Green ${ }^{\circledR} 488$ conjugate (neutravidin-488); and Dil fluorescent membrane dye (1,1'-dioctadecyl-3,3,3',3'-tetramethylindocarbocyanine perchlorate ('DiI'; DiIC18(3))) (DiI). Ultrapure 18.2 $\mathrm{M} \Omega \mathrm{cm}$ water (DI Water) was obtained with the MilliQ Millipore system. Other reagents were purchased from Fisher Scientific: chloroform, sucrose, glucose, and DPBS Buffer $1 \times$ w/o calcium chloride and w/o magnesium chloride (PBS). All lipids purchased were already dissolved in chloroform except Chol which was purchased as powder and subsequently dissolved in chloroform. All dyes and proteins were purchased as powder; the dyes were later dissolved in chloroform and the proteins later dissolved in PBS. The lipid and dye solutions were stored in screw-cap vials at $-20{ }^{\circ} \mathrm{C}$ and the protein solutions were stored at either $-20{ }^{\circ} \mathrm{C}$ or, for immediate use, at $4{ }^{\circ} \mathrm{C}$.

\section{Methods}

\section{Giant vesicle formation}

Giant unilamellar vesicles (GUVs) were electroformed ${ }^{27}$ as follows. Lipids and dyes were mixed at desired proportions, using a Hamilton syringe, to produce a stock solution with net concentration $\sim 10-15 \mathrm{mg} \mathrm{mL} \mathrm{mL}^{-1}$. $5 \mu \mathrm{L}$ of stock solution was deposited to form a thin film on the conductive side of clean indium-tin-oxide (ITO)-coated glass slides (Sigma Aldrich \#703192 8-12 $\Omega \mathrm{sq}^{-1}$ ). To achieve a thin film, a $5 \mu \mathrm{L}$ drop was deposited at one side of the ITO slide and then the syringe needle was used to quickly sweep the drop across the entire slide. Coated slides were shielded from light and held under vacuum with desiccant at approximately $0.1 \mathrm{MPa}$ below atmospheric pressure for $1 \mathrm{~h}$ or more to remove any residual chloroform from the film. Once the vacuum was released, the lipid-coated ITO slides were assembled into a parallel-plate capacitor consisting of two ITO slides facing each other, spaced by a gasket with a narrow opening at the top. Small strips of copper tape were sandwiched between the gasket and the ITO slides on either side to act as electrical leads and the chamber was held together by binder clips. After assembly, the chamber was filled with the desired swelling solution and the gap in the gasket was covered with modelling clay (Sculpey ${ }^{\mathrm{TM}}$ Oven Bake Clay). During the coating, vacuuming, and assembling processes, the lipid film contacted room air at standard pressure for approximately 1 minute maximum total time. This was done to avoid oxidation of the lipids. The assembled chamber was put into an oven at $\sim 60{ }^{\circ} \mathrm{C}$, which is well above the chain-melting temperature of the lipid species used. A function generator was then connected across the two copper leads and an AC voltage applied in three stages. We measure voltage using its root-mean-squared (rms) value. First, the voltage function was set to a $10 \mathrm{~Hz}$ sine wave at $0.05 \mathrm{~V}$ and was increased in increments of $0.05 \mathrm{~V}$ in $3 \mathrm{~min}$ intervals until reaching $0.50 \mathrm{~V}$; second, the $10 \mathrm{~Hz}, 0.50 \mathrm{~V}$, sine wave setting was held for $2 \mathrm{~h}$; third, the function was changed to a $5 \mathrm{~Hz}$ square wave and set to $1.20 \mathrm{~V}$ and held for $30 \mathrm{~min}$. After completing the electroformation process, the function generator and the oven were turned off and the GUVs were allowed to cool to room temperature in situ for about $1 \mathrm{~h}$. The cooled GUVs were gently removed from the chamber using an 18-gauge syringe needle, transferred to an Eppendorf tube, and diluted with 0.75-1.00 $\mathrm{mL}$ of room-temperature swelling solution.

\section{Small vesicle formation}

Small vesicles (SVs) were formed by extrusion ${ }^{28}$ as follows. Lipids and dyes were mixed in a test tube at desired proportions, using a Hamilton syringe, to a net concentration of $\sim 10-25 \mathrm{mg} \mathrm{mL}^{-1}$. The solution was dried by swirling in the bottom of the tube under a light stream of flowing nitrogen. This left a film of dry lipid on the walls of the test tube. The lipid-coated tube was shielded from light and held under vacuum with desiccant at approximately 0.1 MPa below atmospheric pressure for $1 \mathrm{~h}$ or more to remove any residual chloroform from the film. $500 \mu \mathrm{L}$ of DI water was added to the test tube, which was then vortexed until all the visible lipid was suspended in the water. The lipid suspension was then extruded in two steps using an Avani ${ }^{\circledR}$ Mini-Extruder \#610000: first, $250 \mu \mathrm{L}$ of the solution was filtered 10 times through a $0.4 \mu \mathrm{m}$-pore membrane (Whatman Nucleopore $19 \mathrm{~mm} \# 800282)$; second, the same solution was filtered 10 times through a $0.03 \mu \mathrm{m}$-pore membrane (Whatman Nucleopore $19 \mathrm{~mm} \# 800307)$. The SV solution was diluted $2 \times$ with PBS and was used to form supported lipid bilayers (as described below) within 12 hours.

\section{Sample preparation}

To prepare substrates for supported lipid bilayers (SLBs), we cleaned Menzel-Glaser $24 \times 32 \mathrm{~mm}$ \#1 cover glasses using Piranha etching, ${ }^{28}$ using 3:1 98\% sulfuric acid: $30 \%$ hydrogen peroxide. 
This made the glass surfaces very hydrophilic. Cleaned cover glasses were stored in a container filled with DI water until they were used for an experiment; cover glasses were used no more than two days after Piranha etching. For all experiments, two sample slides were prepared in parallel. One sample served as the control, in which GUVs sedimented but did not specifically adhere to the SLB. For this sample, we made an SLB as described below, but did not functionalize the SLB with neutravidin. The other sample was the test sample in which GUVs specifically adhered to the SLB via biotin-avidin binding. This SLB was prepared as described below and then functionalized with neutravidin.

We dried cover glasses using a stream of nitrogen gas, placed a rubber gasket in the centre of each glass, and sealed the gasket's edges with vacuum grease. To make SLBs, $200 \mu \mathrm{L}$ of the SV + PBS buffer mixture was added to the centre of each gasket and allowed to sit for 30 minutes before the excess SVs were rinsed from the glass. We rinsed the SVs by adding another $200 \mu \mathrm{L}$ of PBS buffer inside the gasket, pipetting back up, and discarding. This was repeated 15 times. Then, $50 \mu \mathrm{L}$ of neutravidin at $\left(75 \mu \mathrm{g} \mathrm{mL} \mathrm{m}^{-1}\right)$ was added to the designated test SLB and allowed to sit for 45 minutes. The test SLB was then rinsed, as before, and $50 \mu \mathrm{L}$ of the prepared GUV suspension was added. The sample was then capped with a $22 \times 22$ cover glass and allowed to sit for at least 10 minutes to allow time for the GUVs to adhere. The control slide was prepared similarly, except that it was not functionalized with neutravidin.

To control the pre-adhesion tension in the GUV membranes, we tuned the sucrose solution to be iso-osmolar with the PBS.

\section{Microscopy temperature control and data analysis}

Membranes were imaged by epifluorescent microscopy on an Olympus IX71 inverted microscope at $100 \times$ magnification with an Olympus TIRF UIS2 UAPON objective and by confocal microscopy on an Olympus IX81 inverted microscope at $100 \times$ magnification with an Olympus UPlanFl N objective. Temperatures were controlled using a Physitemp TS-4 SPD Controller. When samples were heated, the temperature reading was calibrated to a measured temperature calibration curve which we constructed by inserting a thermocouple into one of our sample chambers and measuring the sample temperature as a function of the controller's temperature reading. Data analysis was done in ImageJ and Matlab.

\section{Results}

\section{Adhesion induces the formation of an ordered phase}

We chose a 29:29:42 DPPC:DOPC:Cholesterol mixture for this study because previous work has reported that, at room temperature, GUVs of this composition are in a well-mixed, liquid-ordered $\left(\mathrm{L}_{\mathrm{o}}\right)$ lipid phase, close to a phase boundary defining a region where $\mathrm{L}_{\mathrm{o}}$ and liquid-disordered $\left(\mathrm{L}_{\mathrm{d}}\right)$ phases coexist. ${ }^{8}$ In three separate experiments, GUVs that included $1 \mathrm{~mol} \%$ biotin-DOPE, $1 \mathrm{~mol} \%$ PEG-DOPE, and $0.2 \mathrm{~mol} \%$ DiI were electroformed and the resulting yield was split, so that some were added to neutravidin-functionalized SLBs and some to non-functionalized SLBs. The vesicles used to form the SLBs contained $98 \mathrm{~mol} \%$ DOPC, $1 \mathrm{~mol} \%$ biotin-DOPE, and $1 \mathrm{~mol} \%$ PEG-DOPE. DOPC's chain-melting transition temperature is about $-20{ }^{\circ} \mathrm{C}$, so the supported bilayers remained fluid throughout our studies. We verified fluidity by checking that SLBs containing Bodipy recovered after photobleaching; bilayers also appeared continuous. GUVs that were added to neutravidin-functionalized SLBs adhered specifically to the substrate via biotin-avidin binding. GUVs that were added to the non-functionalized SLBs sedimented because of the density difference between internal sucrose solution and external PBS buffer, but did not specifically adhere.

The fluorescent membrane dye DiI is excluded from orderedphase domains as an impurity, so that phase coexistence between the $L_{d}$ phase and an ordered phase appears as bright and dark patches respectively on vesicles. Representative images of a phase-separated and a homogeneous vesicle are shown as insets in Fig. 1.

Of 341 specifically-adhered vesicles, 160 (47\%) were phase separated and the ordered domains were almost always localized to the adhesion zone. That the ordered phase was localized to the adhesion zone is consistent with a recent theoretical prediction for two-component adhering membranes. ${ }^{29}$ Of 262 sedimented vesicles, $44(17 \%)$ had at least one ordered-phase domain (not necessarily localized to the bottom). These data are summarized in Fig. 1.

To check the significance of these results, we used a binomial probability distribution function $\operatorname{Pr}(x=k)=\frac{n !}{k !(n-k) !} p^{k}(1-p)^{n-k}$ to describe the likelihood that exactly $k$ membranes phase separate out of $n$ trials, with a probability of phase separation $p=0.17$. This probability is taken from the sedimented vesicles' distribution. Using the cumulative distribution function of

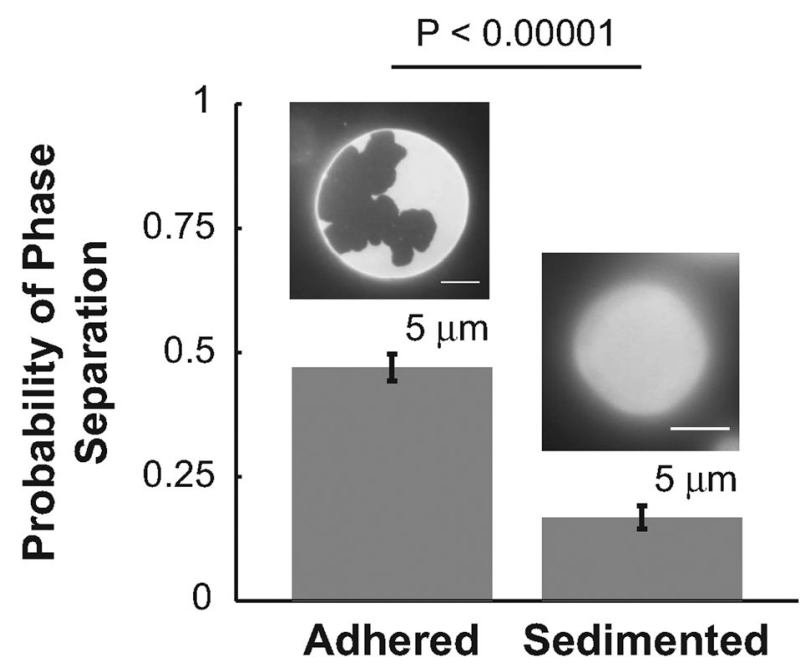

Fig. 1 Specific adhesion promotes the formation of an ordered lipid phase in initially-homogeneous vesicles. Here we show the fractions of membranes that contained an ordered-phase domain for 370 adhered and 262 sedimented vesicles. The error bars are the standard error of the mean by approximating the binomial distributions as Gaussian. Data from three independent experiments are used. Each experiment studied adhering and compositionally-identical sedimented vesicles in parallel. 
the binomial distribution, we estimate the probability of more than $159=k$ vesicles separating after $341=n$ trials as $\operatorname{Pr}(x>k)=1-\sum_{i=0}^{k} \frac{n !}{i !(n-i) !} p^{i}(1-p)^{n-i}<0.00001$.

We conclude that specific adhesion significantly increases the probability that $\mathrm{L}_{\mathrm{d}}$ lipid membranes will phase separate to form an ordered phase. Since the specifically-adhering and sedimented vesicles are otherwise identical in composition and preparation history, we infer that adhesion per se is the cause of the increased likelihood of forming an ordered phase.

We have previously found that non-specific adhesion-in which membranes contact each other directly, without the mediation of binding proteins-can promote the formation of ordered phases in mixed-lipid membranes. ${ }^{11}$ In our earlier work, we suggested that this could result from adhesion suppressing thermally-driven undulations in the membrane. Undulations increase the system's entropy. Forming a stiffer, ordered phase out of the disordered fluid phases $\mathrm{L}_{\mathrm{d}}$ or $L_{\alpha}$ will reduce the amplitude of undulations and is therefore entropically disfavoured. Adhesion pays part of this entropic cost by pre-reducing the amplitude of undulations. Other researchers have examined the role of membrane curvature in the segregation of pre-existing phases. ${ }^{14,30,31}$ They found that stiffer, ordered-phase domains segregate to regions of lower curvature. This minimizes the net bending energy of the system. We have previously estimated that, for spherical membranes of the $\sim 10 \mu$ m radius typifying our GUVs, the effect of undulation suppression will be stronger than that of changes in gross curvature caused by adhesion. ${ }^{11}$ However, in an analysis that does not consider the role of undulations, it has been suggested that adhesion-caused change in gross curvature could lead to the formation of an ordered domain upon adhesion, since the curvature is zero in the adhering region ${ }^{32}$ if the substrate is flat. Both undulation-based and gross curvaturebased interpretations assume that the formation of ordered-phase domains reduces the system's free energy. Therefore, these two interpretations are compatible with but do not necessarily require equilibrium phase separation.

In the following Results subsections, we examine different aspects of adhered, phase-separated membranes to evaluate the degree to which they may be equilibrated and the degree to which they may be non-equilibrated.

\section{The ordered-phase domains are robust to increases in temperature to above the DPPC transition temperature}

Lipid phase separation can result from a quench in temperature that takes a mixture of lipids across a phase boundary. A straightforward equilibrium interpretation of our results would imply that adhesion raised the temperature of the phase boundary, but not above the transition temperature of the highest-melting component of our system. We investigated the temperature response of the ordered phases formed in adhered membranes. The temperature was raised in increments of $2{ }^{\circ} \mathrm{C}$ with 5 minute intervals between to allow the sample to thermally equilibrate. We found that even when the temperature of the sample was as high as $55^{\circ} \mathrm{C}$, the ordered phase domains showed no changes in size or shape. This result is surprising because our system's highest-melting component, the lipid DPPC, has a chain-melting transition temperature of only $41{ }^{\circ} \mathrm{C}$.

We conclude that either these membranes are far from equilibrium, or that adhesion drastically modifies the free energy landscape of the membrane. In our previous work we estimated that suppressing undulations could change the free energy of demixing by about $3 \mathrm{~K}^{11}$ This is inadequate to explain a shift in transition temperature of more than $14 \mathrm{~K}$. However when membranes are bound via biotin-neutravidin, there is a debinding free energy cost of about $10 k_{\mathrm{B}} T$ per biotin-neutravidin bond. ${ }^{19}$ We therefore infer that the ordered-phase domains we see in adhering regions may or may not be in thermodynamic equilibrium.

\section{The geometry of adhering membranes suggests adhered vesicles are in mechanical equilibrium}

Adhesion also modifies the shape of GUVs from their initial sphericity. We used confocal microscopy to measure the 3-dimensional shapes of adhered GUVs (Fig. 2A and B).

Since our GUVs are spherical, to optical microscopy, before adhesion, we compare the measured volume of adhered GUVs with the volume that a sphere with the same surface area would have. Strikingly, we found that adhered vesicles fall into two distinct groups. Not-phase-separated GUVs had volumes $0.975 \pm$ 0.005 that of an equivalent-area sphere, and phase-separated GUVs had volumes $0.838 \pm 0.004$ that of an equivalent-area sphere (Fig. 3A; Fig. S1A, ESI $\dagger$ zooms in on the portion of this plot representing smaller vesicles). From this, we infer that GUVs that phase separated upon adhesion lost more of their internal volume than did GUVs that did not phase separate.

We also measure the proportion of the GUV membrane that was adhered to the substrate by taking the ratio of adhering and non-adhering area for each GUV. Vesicles also fall into two distinct groups for this measurement. Phase-separated membranes have an (adhered area)/(non-adhered) ratio of $0.39 \pm 0.01$, whereas not-phase-separated membranes have an (adhered area)/(nonadhered) ratio of $0.13 \pm 0.02$ (Fig. 3B; Fig. S1B, ESI $\dagger$ zooms in on the portion of this plot representing smaller vesicles). A greater fraction of the membrane has spread onto the flat substrate for GUVs that phase separated upon adhesion than for GUVs that did not phase separate.

Mechanical equilibrium for the adhesion of spherical membranes implies that the adhered area will grow until the reduction of free energy by making one more biotin-neutravidin bond is equal in magnitude to the gain in elastic energy this bond will cause by tensing the membrane. Vesicles losing internal volume, which we find associated with phase separation, is equivalent to vesicles gaining excess membrane area. Taken together, the two sets of observations in Fig. 3 are consistent with our vesicles being in mechanical equilibrium.

\section{A single thermodynamic coordinate governing whether phase separation occurs is insufficient to account for the distribution of ordered-phase domain sizes}

Following the lead of other researchers, we take the miscibility transition temperature to be the temperature at which half 

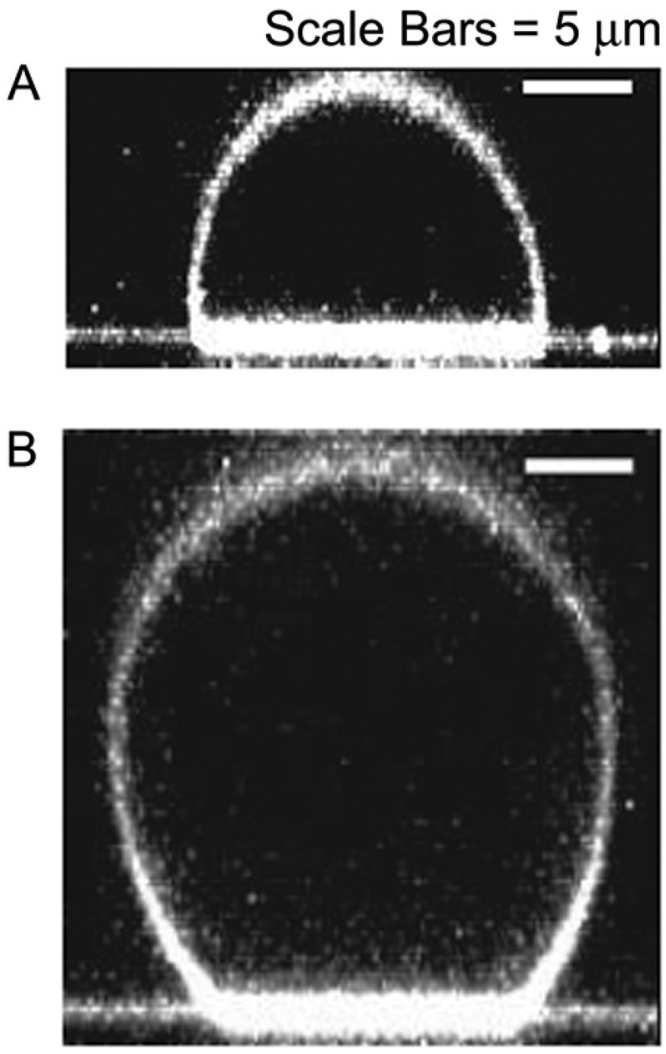

Fig. 2 Adhered vesicles are prolate ellipsoids but phase-separated-vesicle geometry differs from non-phase-separated vesicle geometry. (A) A vertical slice through an adhering, phase-separated vesicle. (B) A vertical slice through an adhering, non-phase-separated vesicle.

the GUV membranes (which will have some variability in their lipid composition) phase separate; for our GUVs, the transition temperature is $\sim 20{ }^{\circ} \mathrm{C}$ in the absence of adhesion. ${ }^{8,10}$ When our membranes adhere, half of them are phase separated at room temperature, which is nearly $24^{\circ} \mathrm{C}$ (Fig. 1).

With only half the adhering vesicles phase separating, a single-coordinate equilibrium view implies that only half the GUVs have an adhesion-shifted demixing transition temperature greater than room temperature. This implies that the high-melting lipid distribution peaks for the lowest composition for which phase coexistence is observed, and that in the coexistence region (grey shading) vesicles with larger highmelting lipid content should occur with decreasing frequency (Fig. 4A).

According to the "lever rule", ${ }^{33}$ the amount of ordered-phase area should grow with the fraction of high-melting lipids. However, this is not what we observe. Instead, we find that the frequency distribution of ordered-phase area fraction is roughly symmetric about an area fraction of $0.22 \pm 0.03$ (Fig. 4B). This suggests that whatever parameters cause an adhered membrane to nucleate an ordered-phase domain are not the same as the parameters that determine the final amount of ordered phase formed.

We infer that either another thermodynamic coordinate is necessary to explain an equilibrium distribution like what we measure, or lipid-driven phase separation is being competed
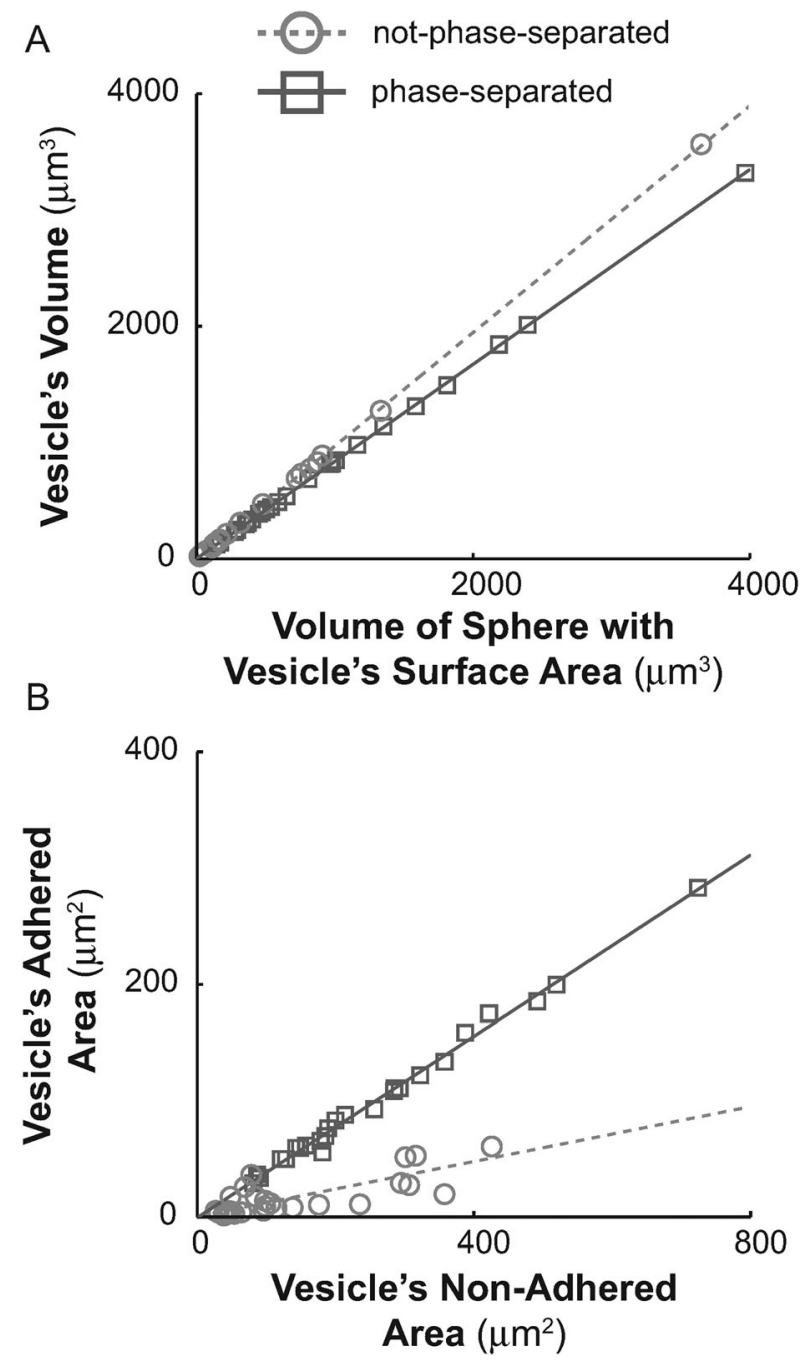

Fig. 3 Adhered vesicles' geometries have two distinct states: one for phase-separated vesicles and another for not-phase-separated vesicles. (A) The ratio of adhered vesicles' volume to the volume of spheres whose surface areas are the same as the vesicles' surface areas. Dark grey squares are phase-separated vesicles with a solid-line fit whose slope is $0.838 \pm$ 0.004 and $R^{2}>0.99$. Light grey circles are not-phase-separated vesicles with a dashed-line fit whose slope is $0.975 \pm 0.005$ and $R^{2}>0.99$. (B) The ratio of adhered vesicles' adhered surface area to the non-adhered surface area. Dark grey squares are phase-separated vesicles with a solid line fit whose slope is $0.39 \pm 0.01$ and $R^{2}>0.99$. Light grey circles are nonphase-separated vesicles with a dashed-line fit whose slope is $0.13 \pm 0.02$ and $R^{2}>0.87$. The reported uncertainties in $(A)$ and $(B)$ are the $95 \%$ confidence intervals.

with by non-equilibrium interactions that originate from the protein binders.

The shapes of ordered-phase domains in adhering membranes are inconsistent with equilibrium separation of fluid lipid phases

Equilibrium thermodynamics can also inform expectations about the shape of phase-separated domains. It is well-established from previous work that fluid-phase domains, whether their phase is liquid-ordered $\left(\mathrm{L}_{\mathrm{o}}\right)$ or liquid-disordered $\left(\mathrm{L}_{\mathrm{d}}\right)$, will have circular 
A

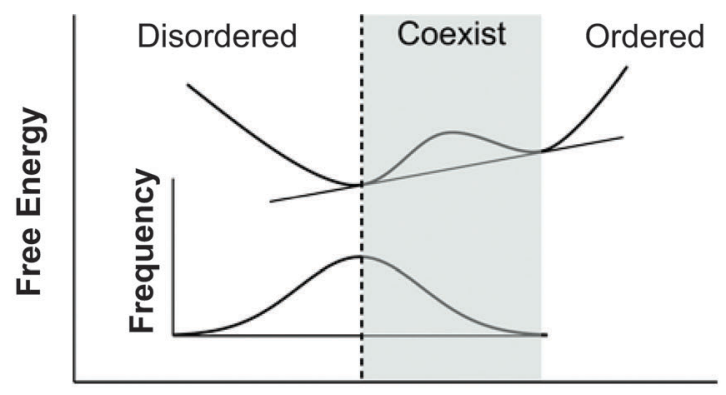

Thermodynamic Coordinate

B

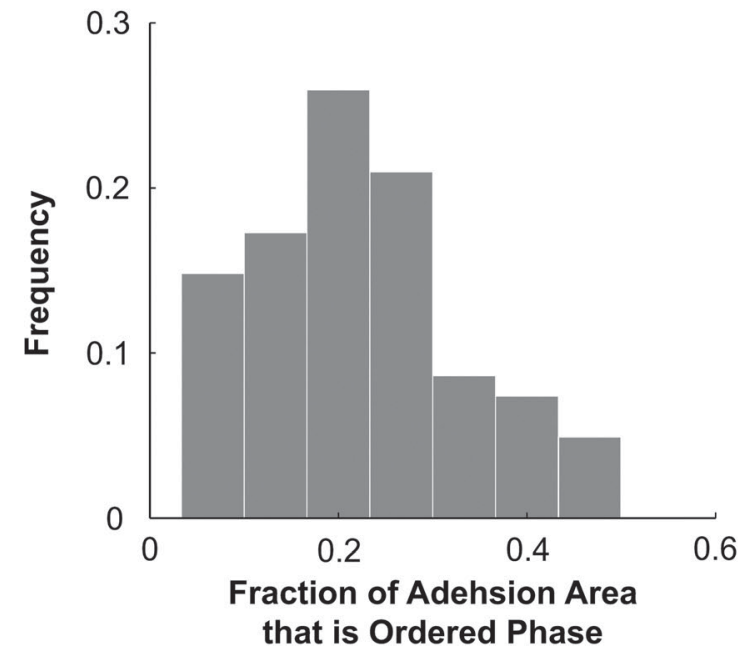

Fig. 4 For specifically-adhering GUVs that phase separate to form ordered-phase domains, the amount of ordered phase present is different than expected for free floating GUVs. (A) GUVs made by electroformation have a variable composition. We sketch the dependence of both the free energy (main plot) and the frequency (inset) of GUVs forming with a particular value of a thermodynamic coordinate determining if a vesicle will phase separate. Half of the GUVs phase separate (indicated by the greyed region; supported by Experimental data in Fig. 1). In the coexistence region (grey shading), the likelihood of measuring a given fraction of ordered phase is a monotonically decreasing function. ${ }^{33}$ (B) In contrast to the schematic in panel $(A)$, the frequency distribution of 81 orderedphase area fractions is roughly symmetric about an area fraction of $0.22 \pm$ 0.03 . This histogram shows aggregated data for three experiments; data broken down by experiment is shown in Fig. S2, ESI. $\dagger$ The reported uncertainty is the standard error of the mean.

shapes at equilibrium. ${ }^{79} \mathrm{~A}$ circular perimeter minimizes the total boundary between phases and thereby minimizes the total line tension and the energy of the system. However, the ordered-phase domains in adhesion zones are not circular (Fig. S3, ESI†). We quantified the circularity of ordered-phase domains using the measure circularity $=4 \pi \frac{\text { area }}{\text { circumference }^{2}}$ which is unity for a circular region and decreases with increasing ratio of perimeter to area, i.e., with increasing departure from a circular shape. Of 83 measured ordered phase domains we found circularity = $0.41 \pm 0.13$.

We conclude that the ordered-phase domains created with specific adhesion do not represent equilibrated $\mathrm{L}_{\mathrm{o}}-\mathrm{L}_{\mathrm{d}}$ lipid phase separation. Either adhesion has modified the equilibrium phase behaviour of our membranes, or these membranes are out of thermodynamic equilibrium.

\section{Ordered-phase domains exclude and are bounded by biotin-neutravidin binding proteins}

To assess the role that biotin-neutravidin binders might play in keeping the membrane out of thermodynamic equilibrium by limiting the growth of ordered-phase domains and/or shaping non-circular domains, we adhered vesicles using neutravidin that was conjugated with the fluorophore Oregon Green (neutravidin-488). Oregon green is spectrally distinct from DiI, which allowed us to separately image the binding proteins and the disordered-phase membrane. In every specifically-adhering vesicle containing an ordered-phase domain, the fluorescent signal from the neutravidin- 488 was excluded from the orderedphase domain and appeared uniform in the disordered region (Fig. 5A-C). Conversely, every vesicle that did not phase separate displayed a uniform fluorescent signal from the neutravidin-488 throughout the adhesion region (Fig. 5D-F).

From this, we conclude the biotin-neutravidin binders are uniformly dense (to optical microscopy) in the disorderedphase portion of the adhering area and that binders are absent from the ordered-phase region. Moreover, we infer that the ordered phase excludes biotinylated lipids bound to neutravidin as impurities similar to its exclusion of the amphiphilic dye DiI.

Impurities, like our binders and dye, can be excluded from ordered lipid phases when their inclusion in the ordered phase costs more free energy than their exclusion to the disordered phase. This is specific to the chemical structure of the impurity and the molecular arrangement of the lipid phases involved. ${ }^{34}$ Thus, partitioning of impurities is consistent with equilibrium physics.

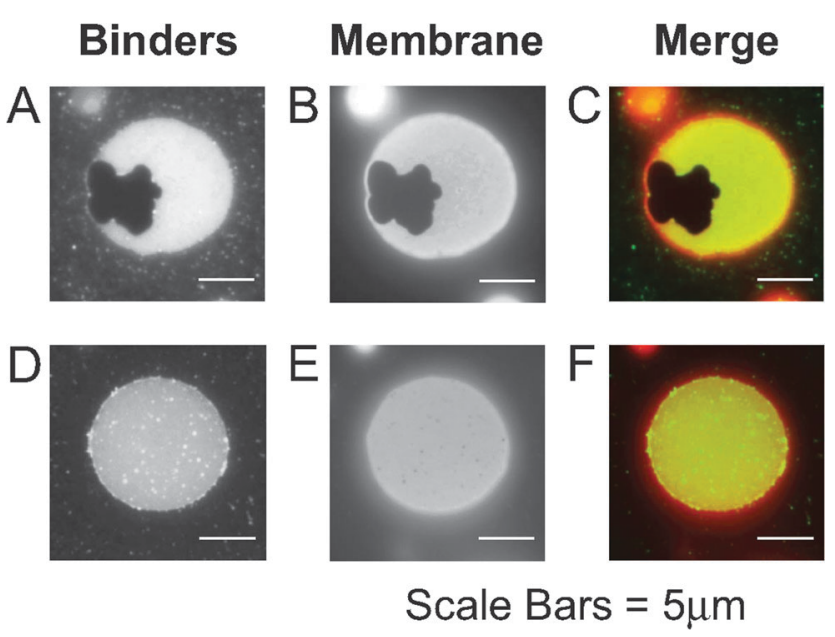

Fig. 5 Biotin-neutravidin binders are excluded from ordered-phase domains and are uniformly distributed in adhering, disordered-phase regions. Epifluorescent micrographs show, (A and D) fluorescentlylabelled neutravidin (Oregon green) and ( $B$ and $E$ ) the fluorescent disordered-phase marker Dil. These two spectrally-distinct channels are co-localized in both phase separated and non-phase separated vesicles as illustrated by false-colour merged images ( $C$ and F). Neutravidin is falsecoloured green and Dil is false-coloured red. 
However binders can jam together and become immobilized; if the binders in the binder-rich, disordered-phase region are kinetically trapped, then our membranes' phase domains may not be in thermodynamic equilibrium.

\section{The disordered-phase marker DiI is enriched in regions with binding proteins}

It has recently been shown that GUV membranes containing the same components as ours, modulo the substitution of DiPhyPC for DOPC as the low-melting-temperature lipid, and streptavidin for neutravidin, will be enriched in the disorderedphase marker DiI in the adhesion zone if the membrane composition is near a critical point. ${ }^{20}$ This enrichment has been attributed to a molecular affinity of the DiI dye, which is a two-tailed lipophile, for the hydrophobic tail regions of the biotinylated DOPE. Our membranes are not near a critical point. To determine whether the binder-containing regions of our membranes are also enriched in DiI, we followed the measurement tactic used in Zhou et al. ${ }^{20}$ and found the ratio of the fluorescent intensity of DiI in the disordered-phase part of the adhesion region to the intensity of DiI at the top of the same vesicle, where the membrane is non-adhering: $\frac{\text { intensity in the disordered }- \text { phase adhering region }}{\text { intensity in the non-adhered vesicle top }}$. This ratio will be greater than one if the adhering region is enriched in DiI.

We account for photobleaching effects by measuring two sets of adhering vesicles in opposite orders - adhering bottom first and top second, or else top first and bottom second. We found that imaging the bottom first produced enrichment measurements greater than if the top was imaged first, so we infer that photobleaching can have a pseudo-enhancing and pseudo-diminishing effect on our measurements. We interpret the geometric average of these two histograms as the approximate real enrichment ratio, which was $1.6 \pm 0.2$ (Fig. 6A). This is similar to that found in Zhou et al. ${ }^{20}$ and indicates that the disordered phase in specifically-adhering regions contains more DiI than does the disordered phase in the free membrane. When we made corresponding measurements on the bottom and top of sedimented vesicles, the real enrichment ratio was $1.0 \pm 0.1$ (Fig. 6B), meaning that there is about the same amount of DiI in the top and bottom of sedimented vesicles.

It is striking that we find DiI enrichment resulting from adhesion in membranes that are not near a critical point, whereas Zhou et al. reported DiI enrichment only in proximity to a critical point, albeit in membranes using DiPhyPC as a lowmelting lipid rather than DOPC. ${ }^{20}$ To check that our measurement techniques yielded results in agreement with Zhou et al. we made GUVs of the same critical composition ${ }^{20}$ (30:20:50 DiPhyPC : DPPC : cholesterol) and found enrichment similar to that reported previously (Table S1, ESI $\dagger$ ). We also checked for enrichment in adhering vesicles made of $21.5: 21.5$ : 57 DPPC: DiPhyPC : cholesterol, which phase separate at room temperature. ${ }^{10}$ Again, we measured DiI enrichments in ordered-phase adhered regions similar to those found at the critical composition (Table S1, ESI $\dagger$ ). When we use fluorescently-labelled neutravidin,
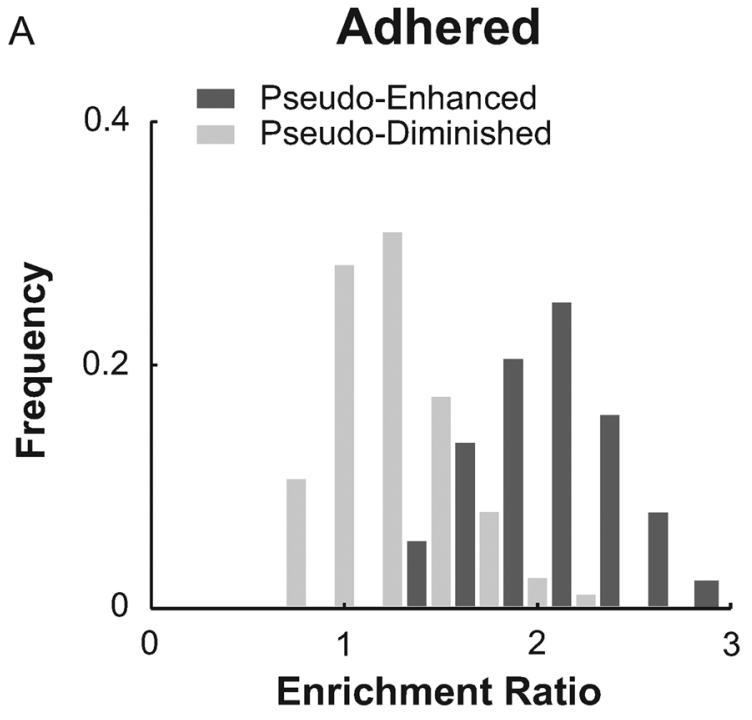

B

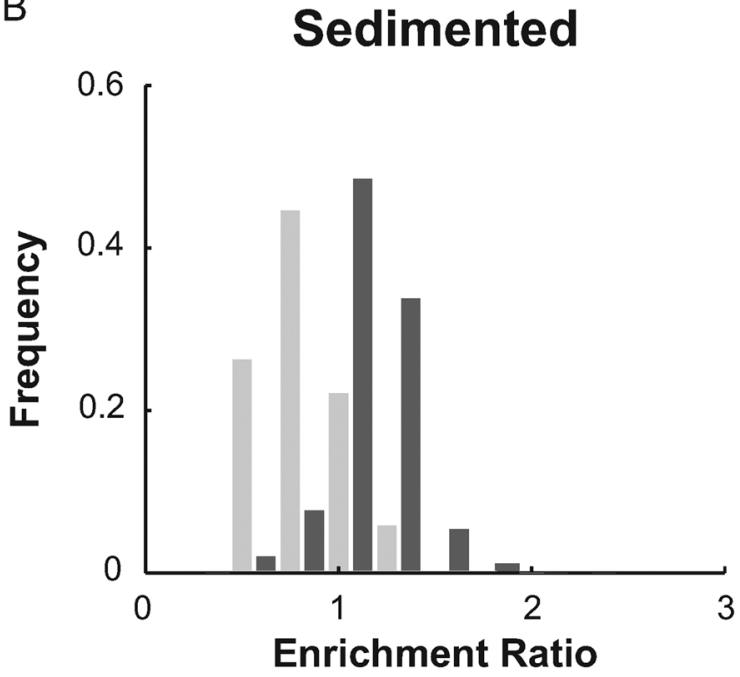

Fig. 6 Specific adhesion enriches the disordered-phase region of the adhering membrane in the membrane dye Dil. Shown are histograms of the ratios of Dil intensity in the disordered-phase adhering region to Dil intensity at the non-adhering top of the membrane. Measuring an enrichment ratio greater than one indicates that the disordered-phase in the adhering region contains a higher density of Dil than the free membrane. To account for photobleaching resulting from fluorescence excitation, we imaged in two orders: bottom and then top resulted in pseudo-enhanced enrichment (dark grey bars); top and then bottom resulted in pseudodiminished enrichment (light grey bars). (A) In specifically-adhered vesicles the average enrichment measurement across the two data sets is $1.6 \pm$ 0.2 ; (B) in sedimented vesicles the average enrichment measurement across the two data sets is $1.0 \pm 0.1$. The reported errors are the standard deviations of the distributions.

we find that DiPhyPC-containing vesicles have the neutravidin fluorescent signal always and only co-localized with the DiI signal (Fig. S4, ESI $\dagger$ ).

From these results we conclude that the disordered-phase regions in the adhesion zones are enriched in membrane dye relative to the non-adhered portion of the vesicle membrane; this enrichment effect is present in both phase-separated and 
non-phase-separated vesicles and is absent in non-adhered, sedimented vesicles.

It is evident that the interface between the GUV and the SLB is enriched in neutravidin compared with the rest of the SLB (Fig. 5A and D). Following Zhou et al., ${ }^{20}$ we attribute the enrichment of dye in the binder-rich portion of the adhering membrane with the molecular affinity of DiI for the tails of biotin-conjugated DOPE lipids. Assuming the DiI molecules are free to diffuse across the boundary between the adhered portion of the membrane and the non-adhered portion of the membrane, the dye enrichment in the adhesion zone is probably thermodynamically equilibrated.

\section{Specific adhesion simultaneously supports dual heterogeneities in lipids and proteins}

All our results taken together demonstrate that adhesion mediated by a single protein interaction results in the formation and stabilization of two types of coexisting domains: (1) an orderedlipid phase that is enriched in the higher-melting lipid and that excludes the DiI membrane dye and biotin-neutravidin binders; and (2) a disordered lipid phase that is enriched in the lower-melting lipid, the DiI membrane dye, and biotin-neutravidin binders. These heterogeneities may or may not be in thermodynamic equilibrium.

\section{Discussion}

The DPPC:DOPC:cholesterol mixture that we have used in our experiments is known, at room temperature, to be near a coexistence region containing fluid-ordered $\left(\mathrm{L}_{\mathrm{o}}\right)$ and fluiddisordered $\left(\mathrm{L}_{\mathrm{d}}\right)$ lipid phases. ${ }^{8}$ It was our expectation upon beginning these experiments that adhesion of homogeneous, $\mathrm{L}_{\mathrm{d}}$ GUVs would result in the formation of circular $\mathrm{L}_{\mathrm{o}}$ domains. However, the ordered-phase domains that we see are notably not circular and they do not change shape over the timescale of a day or with drastic increases in temperature. Therefore, the ordered-phase domains we see in adhering GUVs are not equilibrated $\mathrm{L}_{\mathrm{o}}$ domains, since the line tension at domain perimeters would make a circular $\mathrm{L}_{\mathrm{o}}$ domain the lowest-energy shape for co-existing $\mathrm{L}_{\mathrm{d}}-\mathrm{L}_{\mathrm{o}}$ phases. We suggest two possible, but not mutually exclusive, explanations for non-circular domains: (1) the ordered phase may be gel rather than liquid-ordered and/ or (2) the binders are in a jammed state preventing the domains from equilibrating.

(1) Others have suggested that when a vesicle membrane is phase separated into $L_{o}$ and $L_{d}$ phases, increasing the membrane tension may change the ordered phase from liquid-ordered to gel; a mechanism for this has not been shown. ${ }^{13}$ Our membranes are adhesively tensed. If increasing membrane tension causes the liquid-ordered phase to transition to a gel phase, the diffusion in the gel phase will be many orders of magnitude slower than in a liquid phase. ${ }^{35}$ Very slow kinetics could effectively "freeze" the shape of the domain as it was when the gel formed so that no detectible changes happen over $\sim 24$ hours of observation.
An alternative possible pathway for forming gel domains comes from the effect of adhesion on the free energy landscape of the GUV membranes. The free energy landscape of DPPC:DOPC:cholesterol bilayers should contain a gel phase. At the composition and temperature of our experiments, for non-adhered membranes the free energy of the gel phase should lie higher than the free energies of the $\mathrm{L}_{\mathrm{d}}$ and $\mathrm{L}_{\mathrm{o}}$ fluid phases. However, it could be formed as a metastable phase, as we have seen before for lipid membrane systems that undergo a rapid, deep quench by lowering temperature. ${ }^{34,36}$ In the present case, the quench would be a sudden decrease in entropy caused by adhesion, rather than a decrease in temperature.

Furthermore, the observation that adhesion favours the formation of ordered phases implies that adhesion will increase the free energy of softer phases more than it will increase the energy of stiffer phases. ${ }^{36}$ Notably, this interpretation relies only on the empirical observation that adhesion favours the formation of ordered phases, and not on any inferred mechanism for how this works. If adhesion increases the free energy of the $\mathrm{L}_{\mathrm{d}}$ and $\mathrm{L}_{\mathrm{o}}$ fluid phases by similar amounts but leaves the free energy of the gel phase relatively unchanged, the commontangent construction of the free energy versus composition curves could give an equilibrium coexistence between $\mathrm{L}_{\mathrm{d}}$ and gel phases rather than $\mathrm{L}_{\mathrm{d}}$ and $\mathrm{L}_{\mathrm{o}}$ phases (Fig. S5, ESI $\dagger$ ).

(2) Binding proteins may hinder the equilibration of lipid phase separation. Here we stress the correspondence between our work and that of Fenz et al., ${ }^{19}$ who used the same specificadhesion system that we do except that their GUV membranes were based on unitary SOPC bilayers rather than our ternary lipid bilayers. Those researchers mapped out how the states of protein distribution depend on the density of binders in the GUV and in the SLB and found that at high binder concentrations in the SLB, i.e., $0.5 \%$ or greater, the adhesion was complete and the adhesion zones were homogeneously dense in binding proteins. ${ }^{19}$ However at low binder concentrations in the SLB, i.e., less than $0.5 \%$, adhesion was incomplete and binding proteins were heterogeneously distributed in the adhesion zones. This was found to result in an annulus of high protein density surrounding a protein-free region (see Fig. 2 and 3 of Fenz et al.). ${ }^{19}$ This annulus was attributed to jamming of the proteins; jamming is a non-equilibrium state.

In contrast, the biotin-neutravidin concentrations in our GUVs and SLBs, i.e., $1 \%$ in each, place us well into the complete-adhesion regime described in Fenz et al. ${ }^{19}$ In this regime, binding proteins are homogeneously distributed in the adhesion zone. However, the GUVs in Fenz et al. did not phase separate. We suggest that formation of an ordered phase that excludes binding proteins will exert a lateral pressure and jam binders into even higher concentrations. Jamming will prevent mobility of the binding proteins, which could provide a mechanical restriction that prevents the ordered-phase domains from rounding into circles.

Because biotinylated lipids have DOPE tails, and because the unsaturated DO tails will tend to be in the disordered phase, we surmise that binders may also prevent the area fraction of ordered lipid phase from growing to completion, as discussed 
for Fig. 4. Once the binder density in the adhered region is high, we do not know the degree of lipid-compositional coupling between the adhered and free portions of the same membrane. However, since the fraction of each GUV that is adhered is constant, whether or not the adhered region is compositionally coupled with the non-adhered membrane should not change our equilibrium expectations, based on the lever rule. We suggest that the variation in ordered-phase area fractions that we measure (Fig. 4B) may arise from variable kinetics of binder jamming competing with lipid phase separation and preventing lipid phase separation from maturing to equilibrium during adhesion.

When living immune cells, T-cells and B-cells, adhere to a target they form a stable structure called the immunological synapse that is required for immunity-associated functions. ${ }^{21-23}$ The spatial organization of the mature immunological synapse consists of concentric regions: a central region which is rich in ordered-phase lipids and TCR/MHCp binding complexes preferential to the ordered phase, and a peripheral region rich in disordered-phase lipids and LFA-1/ICAM-1 binding complexes preferential to the disordered phase. The TCR/MHCp binding complex gives an intermembrane separation of $\sim 15 \mathrm{~nm}$ and the LFA-1/ICAM-1 gives an intermembrane separation of $\sim 40 \mathrm{~nm}^{24}$ What interactions give rise to the structure of the immunological synapse is unknown. Theoretical models that combine protein reaction kinetics with the free energy of a fluid lipid membrane can lead to stable heterogeneities in protein distributions in the immunological synapse. ${ }^{25,37-39}$ Having binders of two different sizes was essential for these models to produce stable heterogeneities; binder size impacted the dependence of association kinetics on intermembrane distance and it gave rise to a membrane bending energy cost when unlike complexes were next to each other. These models did not incorporate heterogeneities in lipid distribution or phase.

The topological characteristics of phase separation in our reductionist model system are very nearly those of the immune synapse, with a few exceptions. Our GUVs are made without proteins that are preferential for the ordered phase, and the binder densities in our system are much higher than those in immunological synapses. Most notably, and unlike previous reductionist descriptions, ${ }^{25,37-39}$ we have only one type of binding complex and our GUV membranes are made of a mixture of lipids that can phase separate. Our results show that adhesion itself, even in a highlyreductionist system with only one species of binding complex and only three lipid species, may interplay with lipid phase separation to give rise to nested, dual heterogeneities if the membrane is sufficiently near a phase transition. This suggests that the many different interactions possible for the biological system, resulting from multiple types of binding complexes, cytoskeletal transport, ${ }^{40}$ and much greater multiplicity of lipid species, may have greater fine-tuning power than previously suspected.

\section{Conclusions}

The broad goal of biophysical work on artificial and plasma membrane vesicles is to gain physical insight into the mechanisms driving organization in real cells' membranes. Our work has uncovered an effect leading to heterogeneities at the adhesion site of an artificial two-membrane junction. We show that a single type of adhesive interaction can simultaneously stabilize two types of heterogeneity in membrane protein and lipid composition. The resulting heterogeneities are robust against changes in temperature and represent long-lived non-equilibrium states.

\section{Acknowledgements}

This work was funded by start-up funds from The University of Texas at Austin (UT Austin) to VDG. MR was supported in part by undergraduate research fellowships from UT Austin. We are grateful to Professor Jeanne Stachowiak (Biomedical Engineering, UT Austin) for helpful conversations about membrane formation and to her and her group for technical assistance. We thank Professor Ernst-Ludwig Florin (Physics, UT Austin) for the extruder and for cover glasses. We thank Professor Lauren Ehrlich (Molecular Biosciences, UT Austin) for helpful conversations about the immune synapse.

\section{Notes and references}

1 K. Simons and E. Ikonen, Functional rafts in cell membranes, Nature, 1997, 387, 569-572.

2 M. Edidin, The state of lipid rafts: from model membranes to cells, Annu. Rev. Biophys. Biomol. Struct., 2003, 32, 257-283.

3 K. Simons and W. L. C. Vaz, Model systems, lipid rafts, and cell membranes, Annu. Rev. Biophys. Biomol. Struct., 2004, 33, 269-295.

4 L. J. Pike, Rafts defined: a report on the Keystone Symposium on Lipid Rafts and Cell Function, J. Lipid Res., 2006, 47, 1597-1598.

5 J. F. Hancock, Lipid rafts: contentious only from simplistic standpoints, Nat. Rev. Mol. Cell Biol., 2006, 7, 456-462.

6 D. Lingwood and K. Simons, Lipid rafts as a membraneorganizing principle, Science, 2010, 327, 46-50.

7 S. Veatch and S. Keller, Organization in Lipid Membranes Containing Cholesterol, Phys. Rev. Lett., 2002, 89, 268101.

8 S. L. Veatch and S. L. Keller, Separation of liquid phases in giant vesicles of ternary mixtures of phospholipids and cholesterol, Biophys. J., 2003, 85, 3074-3083.

9 T. Baumgart, S. Hess and W. Webb, Imaging coexisting fluid domains in biomembrane models coupling curvature and line tension, Nature, 2003, 425, 821-824.

10 S. L. Veatch, K. Gawrisch and S. L. Keller, Closed-loop miscibility gap and quantitative tie-lines in ternary membranes containing diphytanoyl PC, Biophys. J., 2006, 90, 4428-4436.

11 V. D. Gordon, M. Deserno, C. M. J. Andrew, S. U. Egelhaaf and W. C. K. Poon, Adhesion promotes phase separation in mixed-lipid membranes, Europhys. Lett., 2008, 84, 48003.

12 T. Portet, S. E. Gordon and S. L. Keller, Increasing membrane tension decreases miscibility temperatures; An experimental demonstration via micropipette aspiration, Biophys. J., 2012, 103, L35-L37. 
13 T. Hamada, Y. Kishimoto, T. Nagasaki and M. Takagi, Lateral phase separation in tense membranes, Soft Matter, 2011, 7, 9061.

14 R. Parthasarathy, C. Yu and J. T. Groves, Curvaturemodulated phase separation in lipid bilayer membranes, Langmuir, 2006, 22, 5095-5099.

15 A. Albersdörfer, T. Feder and E. Sackmann, Adhesioninduced domain formation by interplay of long-range repulsion and short-range attraction force: a model membrane study, Biophys. J., 1997, 73, 245-257.

16 A. Kloboucek, A. Behrisch, J. Faix and E. Sackmann, Adhesion-induced receptor segregation and adhesion plaque formation: a model membrane study, Biophys. J., 1999, 77, 2311-2328.

17 A. Boulbitch, Z. Guttenberg and E. Sackmann, Kinetics of membrane adhesion mediated by ligand-receptor interaction studied with a biomimetic system, Biophys. J., 2001, 81, 2743-2751.

18 S. F. Fenz, R. Merkel and K. Sengupta, Diffusion and Intermembrane Distance: Case Study of Avidin and E-Cadherin Mediated Adhesion, Langmuir, 2009, 1074-1085.

19 S. F. Fenz, A.-S. Smith, R. Merkel and K. Sengupta, Intermembrane adhesion mediated by mobile linkers: effect of receptor shortage, Soft Matter, 2011, 7, 952-962.

20 J. Zhao, J. Wu and S. L. Veatch, Adhesion stabilizes robust lipid heterogeneity in supercritical membranes at physiological temperature, Biophys. J., 2013, 104, 825-834.

21 A. Grakoui, S. K. Bromley, C. Sumen, M. M. Davis, A. S. Shaw, P. M. Allen and M. L. Dustin, The Immunological Synapse: A Molecular Machine Controlling T Cell Activation, Science, 1999, 285, 221-227.

22 S. K. Bromley, W. R. Burack, K. G. Johnson, K. Somersalo, T. N. Sims, C. Sumen and M. M. Davis, et al., The immunological synapse, Annu. Rev. Immunol., 2001, 19, 375-396.

23 H. W. Sohn, P. Tolar and S. K. Pierce, Membrane heterogeneities in the formation of B cell receptor-Lyn kinase microclusters and the immune synapse, J. Cell Biol., 2008, 182, 367-379.

24 M. L. Dustin and J. A. Cooper, The immunological synapse and the actin cytoskeleton: molecular hardware for $\mathrm{T}$ cell signaling, Nat. Immunol., 2000, 1, 23-29.

25 S. Y. Qi, J. T. Groves and A. K. Chakraborty, Synaptic pattern formation during cellular recognition, Proc. Natl. Acad. Sci. U. S. A., 2001, 98, 6548-6553.

26 A. I. Norman, B. A. Manvilla, E. L. Frank, J. N. Niamke, G. D. Smith and S. C. Greer, Partitioning of poly(ethylene oxide), poly(ethylene imide), and bovine serum albumin in isobutyric acid + water, Macromolecules, 2008, 41, 997-1008.

27 M. I. Angelova and D. S. Dimitrov, Liposome electroformation, Faraday Discuss. Chem. Soc., 1986, 81, 301-311.

28 P. M. Nair, K. Salaita, R. S. Petit and J. T. Groves, Using patterned supported lipid membranes to investigate the role of receptor organization in intercellular signaling, Nat. Protoc., 2011, 6, 523-539.

29 T. Rouhiparkouhi, T. R. Weikl, D. E. Discher and R. Lipowsky, Adhesion-induced phase behavior of two-component membranes and vesicles, Int. J. Mol. Sci., 2013, 14, 2203-2229.

30 B. Rózycki, T. R. Weikl and R. Lipowsky, Stable patterns of membrane domains at corrugated substrates, Phys. Rev. Lett., 2008, 100, 098103.

31 M. I. Hoopes, R. Faller and M. L. Longo, Lipid domain depletion at small localized bends imposed by a step geometry, Langmuir, 2011, 27, 2783-2788.

32 Y. Zhao, S. Das and Q. Du, Adhesion of multicomponent vesicle membranes, Phys. Rev. E: Stat., Nonlinear, Soft Matter Phys., 2010, 81, 041919.

33 H. B. Callen, Thermodynamics \& an Intro. to Thermostatistics, John Wiley \& Sons, 2006.

34 V. D. Gordon, P. A. Beales, Z. Zhao, C. Blake, F. C. MacKintosh, P. D. Olmsied, M. E. Cates, S. U. Egelhaaf and W. C. K. Poon, Lipid organization and the morphology of solid-like domains in phase-separating binary lipid membranes, J. Phys.: Condens. Matter, 2006, 18, L415-L420.

35 M. B. Schneider, W. K. Chan and W. W. Webb, Fast diffusion along defects and corrugations in phospholipid P beta, liquid crystals, Biophys. J., 1983, 43, 157-165.

36 V. D. Gordon, et al., Solid-Like Domains in Mixed Lipid Bilayers: Effect of Membrane Lamellarity and Transition Pathway, Adv. Planar Lipid Bilayers Liposomes, Elsevier Ltd, 2014, vol. 20, pp. 137-153.

37 S.-J. E. Lee, Y. Hori and A. K. Chakraborty, Low T cell receptor expression and thermal fluctuations contribute to formation of dynamic multifocal synapses in thymocytes, Proc. Natl. Acad. Sci. U. S. A., 2003, 100, 4383-4388.

38 S. Raychaudhuri, A. K. Chakraborty and M. Kardar, Effective membrane model of the immunological synapse, Phys. Rev. Lett., 2003, 91, 208101.

39 T. R. Weikl and R. Lipowsky, Pattern formation during T-cell adhesion, Biophys. J., 2004, 87, 3665-3678.

40 M. L. Dustin and A. S. Shaw, Costimulation: Building an Immunological Synapse, Science, 1999, 283, 649-650. 